\title{
Recent results of Synchrotron radiation induced Total Reflection X-ray Fluorescence Analysis at HASYLAB, Beamline $\mathbf{L}$
}

\author{
C.Streli ${ }^{1}$, G.Pepponi ${ }^{2}$, P. Wobrauschek ${ }^{1}$, C.Jokubonis ${ }^{1}$, G.Falkenberg ${ }^{3}$, G.Zaray ${ }^{4}$, \\ J.Broekaert $^{5}$, U.Fittschen ${ }^{5}$, B.Peschel ${ }^{5}$ \\ ${ }^{1}$ Atominstitut, Vienna Univ. of Technology, Stadionallee 2, A-1020 Wien, Austria \\ ${ }^{2}$ ITC-irst, Povo, Italy \\ ${ }^{3}$ Hamburger Synchrotronstrahlungslabor at Deutsches Elektronen-Synchrotron DESY, Notkestr. 85, D- \\ 22603 Hamburg, Germany \\ ${ }^{4}$ Inst. of Inorganic and applied Chemistry, 3 EÖTVÖS Univ , Budapest, Hungary \\ ${ }^{5}$ Inst. Of Anorganic and Applied Chemistry, Univ. Hamburg, Martin-Luther-King-Platz 6, 20146 Hamburg, \\ Deutschland
}

At HASYLAB, Beamline $\mathrm{L}$ a vacuum chamber for SR-TXRF analysis is now available which can easily be installed using the adjustment components for microanalysis present at this beamline. The detector is now in the final version a VORTEX Silicon Drift Detector (SDD) with $50 \mathrm{~mm}^{2}$ active area from Radiant Detector Technologies. With the Ni/C multilayer monochromator set to $17 \mathrm{keV}$ extrapolated detection limits of $8 \mathrm{fg}$ were obtained using the $50 \mathrm{~mm}^{2}$ SDD with $1000 \mathrm{~s}$ live time on a sample containing $100 \mathrm{pg}$ of Ni.

Various applications are presented, especially of samples which are available in very small amounts: As SR-TXRF is much more sensitive than tube excited TXRF the sampling time of aerosol samples can be diminished resulting in a more precise time resolution of atmospheric events. Aerosols, directly sampled on Si reflectors in an impactor were investigated. A further application was the determination of contamination elements in a slurry of high purity $\mathrm{Al}_{2} \mathrm{O}_{3}$. No digestion is required, the sample is pipetted and dried before analysis. A comparison with laboratory TXRF showed the higher sensitivity of SRTXRF, more contamination elements could be detected. Using the Sil11 crystal monochromator also available at beamline L, XANES measurements to determine the chemical state were performed. This is only possible with lower sensitivity as the flux transmitted by the crystal monochromator is about a factor of 100 lower than that transmitted by the multilayer monochromator. Preliminary results of XANES measurements for As in xylem sap from cucumber plants fed with AsIII and AsV are reported. Detection limits of $170 \mathrm{ppt}$ of As in xylem sap were achieved.

KEYWORD: SRTXRF, synchrotron radiation, XANES, aerosols, 


\section{INTRODUCTION}

As the outstanding features of synchrotron radiation such as high flux, continuous energy spectrum, natural collimation, linear polarization are perfect prerequisites for TXRF, Synchrotron Radiation induced Total Reflection X-ray Fluorescence Analysis (SR-TXRF) allows the determination of ultra trace elements in various kinds of samples from the environment, medicine and (semiconductor-) industry $[1,2]$ at concentration levels below $\mathrm{ng} / \mathrm{g}$. As only a few $\mu \mathrm{l}$ or some ng of sample are required SR-TXRF is especially suitable for samples where only small amounts (volumes) are available. Using a multilayer for monochromatization, detection limits in the low fg range can be achieved for medium $\mathrm{Z}$ elements[3]. The simple quantification procedure for all elements present referring to an internal standard is another advantage of TXRF.

A vacuum chamber SR-TXRF setup has been installed at HASYLAB, DESY, Hamburg, beamline L- a bending magnet beamline [4]. This setup uses the translation and rotation stages of the regular microfocus setup available at this beamline for adjustment of total reflection. The chamber is equipped with an automatic sample loader for $30 \mathrm{~mm}$ diameter quartz reflectors for inserting the prepared sample reflectors in the measuring position with high reproducibility. Alternatively, a chuck can be inserted allowing various sizes and shapes of samples to be measured. The first experiments have been performed using a 5 $\mathrm{mm}^{2}$ Silicon Drift detector. Detection limits of $15 \mathrm{fg}$ have been achieved using $10 \mathrm{keV}$ monochromatic excitation from a double multilayer monochromator. Using the Si 111 crystal monochromator available at Beamline L, X-ray Absorption Near Edge Structure (XANES) investigations in total reflection geometry on trace elements are also feasible.

\section{EXPERIMENTAL}

A new Silicon Drift Detector (SDD) from Radiant - Vortex [5] with $50 \mathrm{~mm}^{2}$ active area is now available at the beamline. This detector with the corresponding digital pulse processing allows processing of countrates up to $150 \mathrm{kcps}$ with a FWHM of $160 \mathrm{eV}$ with a peaking time of $2 \mu \mathrm{s}$ [6]. For SRTXRF with $17 \mathrm{keV}$ monochromatic excitation radiation, detection limits of $8 \mathrm{fg}$ were obtained on a sample containing $100 \mathrm{pg}$ of $\mathrm{Ni}$ using the $\mathrm{Ni} / \mathrm{C}$ multilayer monochromator. Fig. 1 shows the corresponding spectrum. Extrapolation to 10 $\mathrm{keV}$ excitation energy leads to detection limits of $4 \mathrm{fg}$. 
Further investigations were made to compare two kinds of reflector material, $30 \mathrm{~mm}$ polished flat quartz discs and $30 \mathrm{~mm}$ Si discs prepared from Si wafers with perfect surface quality.

Tab. 1 Comparison of data obtained from a $100 \mathrm{pg}$ Ni sample on a quartz and on a Si wafer sample reflector. Measuring time: $60 \mathrm{~s}$.

\begin{tabular}{ccccc}
\hline & Net counts & Background & LLD(fg) & S(cps/ng/100mA) \\
\hline Quartz & 160800 & 1030 & 14 & 21000 \\
\hline Si wafer & 215000 & 548 & 8 & 35000 \\
\hline
\end{tabular}

The samples prepared on $\mathrm{Si}$ wafers showed lower background than these prepared on quartz reflectors as can be seen from the spectrum in Fig. 2. Tab. 1 shows the net counts obtained, background counts, detection limits and sensitivity. Data evaluation was performed using AXIL [7]: The discrepancy in the sensitivity is a result of the drying process and variations in the dry sample shape; for that reason an internal standard is always required for the quantification process.

\section{RESULTS:}

To investigate the applicability of various sample systems were studied, among aerosols, ultra pure alumina and xylem sap.

Aerosols:

To understand the effect of aerosols on global climate, a detailed understanding of the sources, transport, fate and of the physical and chemical properties of atmospheric particles is necessary. To satisfy this demand, a procedure that provides a size resolution of particles and short sampling times is essential, but these results in very low absolute amounts of collected sample material. This in turn demands highly sensitive detection methods. To optimise sampling and measurements, a procedure that uses the aerosol collector plates of a 12-stage Berner impactor directly as sample carriers for SR-TXRF was developed. Siwafers were chosen as material for these plates. The sensitivity increases remarkably with SR-TXRF compared to a laboratory TXRF instrument using a conventional X-ray tube. This is illustrated by the spectra of atmospheric aerosol particles in the size range of 1-4 $\mu \mathrm{m}$ (aerodynamic particle size) sampled as described above for $1 \mathrm{~h}$ (at $80 \mathrm{~m}$ height, Hamburg) shown in Fig. 3. Instead of 6 elements detected with a conventional TXRF 
equipment (Atomika TXRF 8030C) (Fig. 3 a), 12 elements could be detected with the SRTXRF vacuum chamber (Fig. 3 b). Fig. 4 shows a spectrum of sample from Impactor stage 1: particle diameter smaller than $60 \mathrm{~nm}, 1 \mathrm{~h}$ sampling with excitation energy of $17 \mathrm{keV}$, $100 \mathrm{~s}$ counting time showing a significant $\mathrm{Pb}$ contribution. Improvement in calibration and accuracy in SR-TXRF will be most challenging, as the Berner impactor produces single spots on a sampling stage.

Ultra pure alumina.

$\mathrm{Al}_{2} \mathrm{O}_{3}$ is the most important ceramic-oxide material. Global markets for calcined and tabular alumina have been estimated at around 2 million and 300,000 tons per year, respectively. Ultra pure alumina for example is strongly asked in the area of high performance lighting technology. High pressure sodium (HPS) lamps illuminate the night. As synthetic rubies and yttrium-alumina-garnets (YAG) it is widely used in laser technology. Especially YAG-lasers doped with rare earth elements are real work horses in medical laser applications. With TXRF, elemental impurities in $\mathrm{Al}_{2} \mathrm{O}_{3}$ powders can be determined by using $\mathrm{Al}_{2} \mathrm{O}_{3}$ slurries and without the need for a prior time-consuming digestion of the samples .Fig. 5 shows a comparison of spectra obtained with laboratory TXRF and SR-TXRF. Cr and Mn could only be detected by SR-TXRF. The standard deviation of the SR-TXRF analyses is relatively high, probably due to sample inhomogeneity. The literature values for $\mathrm{Ca}$ and $\mathrm{Zn}$ vary strongly, but the results of both TXRF analysis techniques agree well with each other. [8] SR-TXRF has been shown to provide very good sensitivity which is crucial for the determination of trace elements such as $\mathrm{Cr}$ and $\mathrm{Mn}$ in $\mathrm{Al}_{2} \mathrm{O}_{3}$ powders. The accuracy and precision of these determinations however is limited by the inhomogeneity of the samples, which was found to be problematic for analyses with micro amounts.

Arsenic speciation in xylem sap

The toxicity of arsenic differs considerably as a function of the oxidation state and chemical form. Inorganic As species such as arsenite $[\mathrm{As}(\mathrm{III})]$ and arsenate $[\mathrm{As}(\mathrm{V})]$ are more toxic than organic ones such as monomethyl arsenic (MMA) and dimethyl arsenic (DMA) acids. The LD50 (The LD50 is a standardized measure for expressing and comparing the toxicity of chemicals. The LD50 is the dose that kills half (50\%) of the animals tested ) for humans expressed in $\mathrm{mg} / \mathrm{kg}$ for $\mathrm{As}(\mathrm{III}), \mathrm{As}(\mathrm{V})$, MMA and DMA are 60, 120, 700 and 1600, respectively. Thus, arsenate is less toxic than arsenite. However, plants may have the capability to change the oxidation state of arsenic, therefore, this issue should be investigated. Experiments have been performed by growing cucumber plants in 
hydroponics containing $\mathrm{As}(\mathrm{III})$ or $\mathrm{As}(\mathrm{V})$ as nutrient solutions and to determine the arsenic concentration of the collected xylem saps by SR-TXRF. The As concentration (expected to be in the ppb range) should be determined as well as the oxidation state in different plant parts. The big advantage of SR-TXRF is that the xylem can be directly measured without any sample preparation which could influence the oxidation state and that only small amount of xylem is required for the analysis. Fig. 6 shows XANES spectra from xylem sap, one from a plant feeded with AsIII and one from a plant feeded with AsV. The xylem sap was pipetted directly on a quartz reflector. For comparison, a standard of DMA (dimethylarsenic) was measured. As for this experiment no precise energy calibration was available it was not possible to determine wether it is AsIII or AsV. It could be shown that the oxidation state is the same for both plants. It corresponds to the results of Mihucz et. al [9]. Fig. 7 shows spectrum of xylem sap with an As concentration of about 13 ppb. A 25 $\mu \mathrm{m} \mathrm{Al}$ detector filter had to be used to reduce the countrate of $\mathrm{K}$ and $\mathrm{Ca}$. Detection limits for $30 \mathrm{~s}: 170 \mathrm{ppb}$ As.

So it was possible to show without any sample preparation that speciation on xylem sap is possible resulting in the finding that the oxidation state of As found in xylem sap is independent from the oxidation state of As in the nutrient solution.

\section{CONCLUSIONS:}

A SR-TXRF setup with a vacuum chamber is now available at HASYLAB, Beamline L for all users. This setup is equipped with a $50 \mathrm{~mm}^{2} \mathrm{SDD}$, allowing the processing of countrates up to $100 \mathrm{kcps}$ with acceptable energy resolution. The detection limits obtained for Ni with $17 \mathrm{keV}$ excitation energy are $8 \mathrm{fg}$ extrapolated to $1000 \mathrm{~s}$ measuring time, if the multilayer monochromator is used. The applicability of the setup was demonstrated by analysing impactor samples aerosols, which allows a very short sampling time, analysing clean substances like $\mathrm{Al}_{2} \mathrm{O}_{3}$ which shows more elements than by analyzing with laboratory TXRF. This beamline is also equipped with a Si111 crystal monochromator which enables XANES experiments. Speciation of As have been performed on organic material, with detection limits of $170 \mathrm{ppt}$ for As in $30 \mathrm{~s}$ measuring time.

\section{Acknowledgement:}


The authors would like to thank the FWF ( Austrian Science Foundation ) for financial support under project P 18299

The work was done within the framework of the Hasylab proposal: II-04-06, I-04-089, I03064EC

This work was supported by the European Community - Research Infrastructure Action under FP6 "Structuring the European Research Area" Programme - Integrating Activity on Synchroton and Free Electron Laser Science ().

\section{References:}

[1] P. Wobrauschek, P. Kregsamer, W. Ladisich, C. Streli, S. Pahlke, L. Fabry, S. Garbe, M. Haller, A. Knöchel, M. Radtke, Nucl. Instr. Meth. A363, 619 (1995).

[2] K. Baur, S. Brennan, P. Pianetta, Opila, Anal. Chem., 609A-616A (2002).

[3] R. Rieder, P. Wobrauschek, W. Ladisich, C. Streli, H. Aiginger, S. Garbe, G. Gaul, A. Knöchel, F. Lechtenberg, Nucl. Instr. Meth.A355, 648 (1995).

[4] C.Streli, G.Pepponi,P.Wobrauschek, C.Jokubonis, G.Falkenberg, G.Zaray, X-ray Spectrom. 34,451-455 (2005)

[5] www.radiantdetectors.com/vortex.html

[6] G. Falkenberg, Characterization of a Radiant Vortex Silicon Multi-Cathode X-ray Spectrometer for (total reflection) X-ray fluorescence applications, Hasylab internal Report 2004 ( 2005)

[7] Vekemans B., Janssens, K., Vincze L., Adams, F., Van Espen P., Analysis of X-ray spectra by iterative least squares ( AXIL) : new developments, X-ray spectrometry 23, 278-285 (1994)

[8] B.Peschel, U.Fittschen,G.Pepponi, C.Jokubonis, C.Streli, P.Wobrauschek, G.Falkenberg, J.Broekaert. , Anal Bioanal Chem (2005) 382: 1958-1964

[9] Victor G. Mihucz, Enikö Tatár, István Virág, Edit Cseh, Ferenc Fodor and Gyula Záray, Analytical and Bioanaytical Chemistry 383 (3), 461-466 (2005) 
Figures:

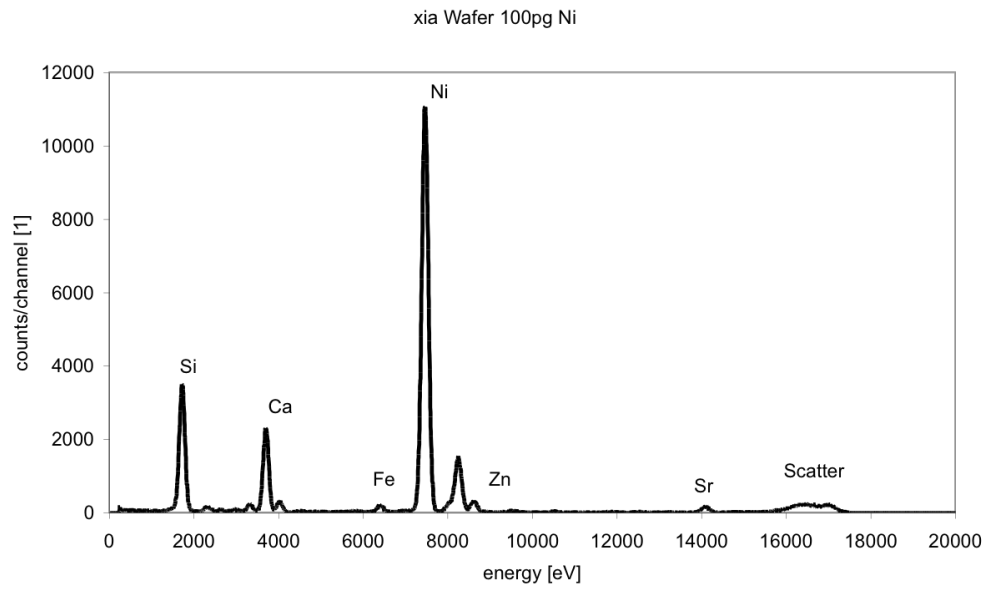

Fig. 1 Spectrum of a sample containing $100 \mathrm{pg}$ of $\mathrm{Ni}$ on a Si wafer as sample reflector excited with $17 \mathrm{keV}$ using the multilayer monochromator, livetime : $60 \mathrm{~s}$, the detection limit was found to be 8 $\mathrm{fg}$, with a sensitivity of $34000 \mathrm{cps} / \mathrm{ng} / 100 \mathrm{~mA}$.

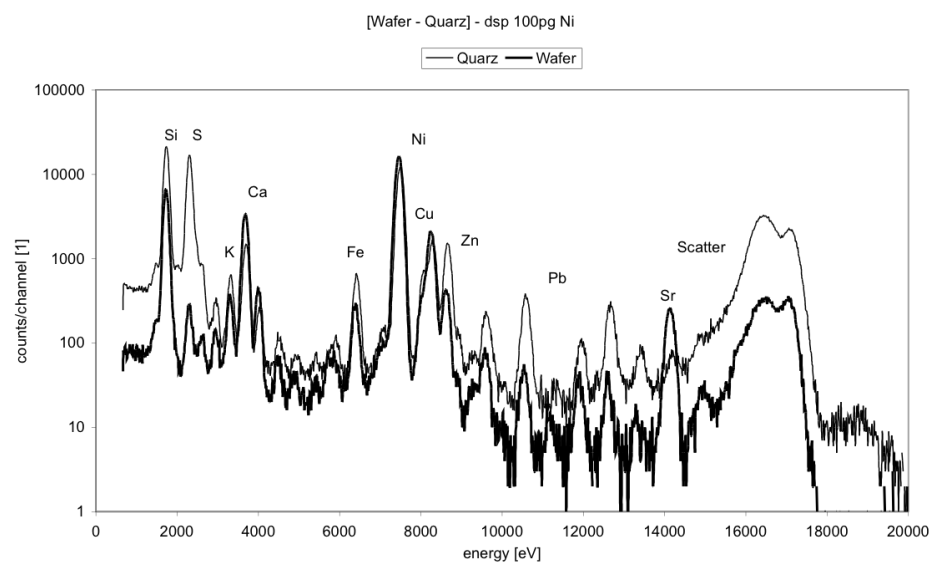

Fig. 2 Comparison of 2 spectra from $100 \mathrm{pg}$ Ni deposited on a Si wafer as well as a quartz reflector showing better background conditions using the Si wafer.
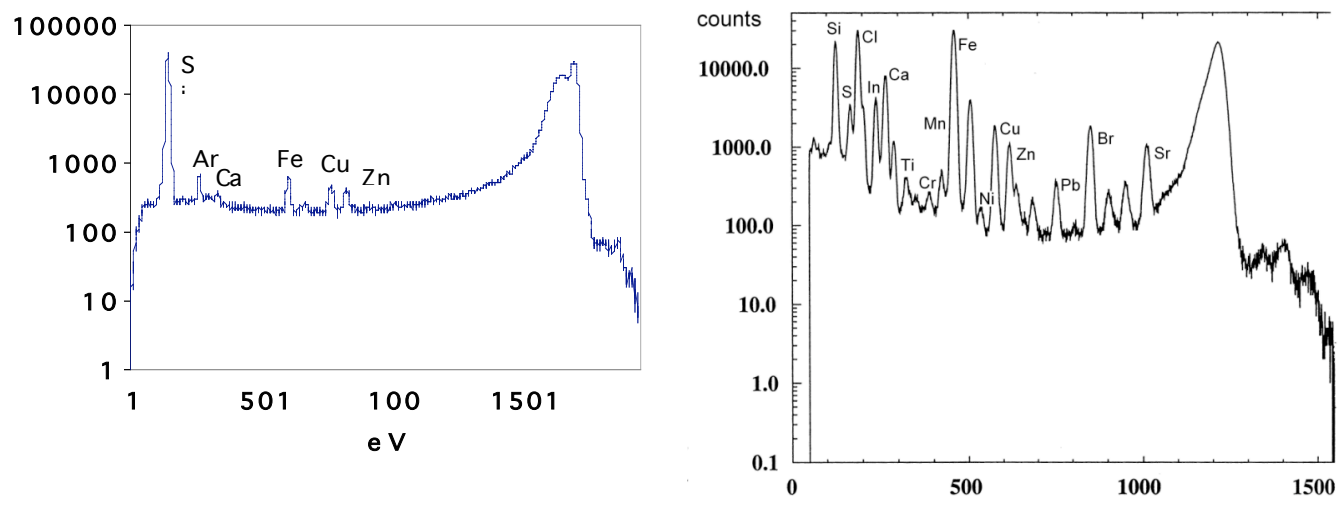

Fig. 3 a) Spectrum of aerosol sample obtained with a conventional TXRF equipment (8030C), measured for $1000 \mathrm{~s}, \mathrm{~b})$ Spectrum of the same aerosol sample obtained with SRTXRF, measuring time $60 \mathrm{~s}$. 


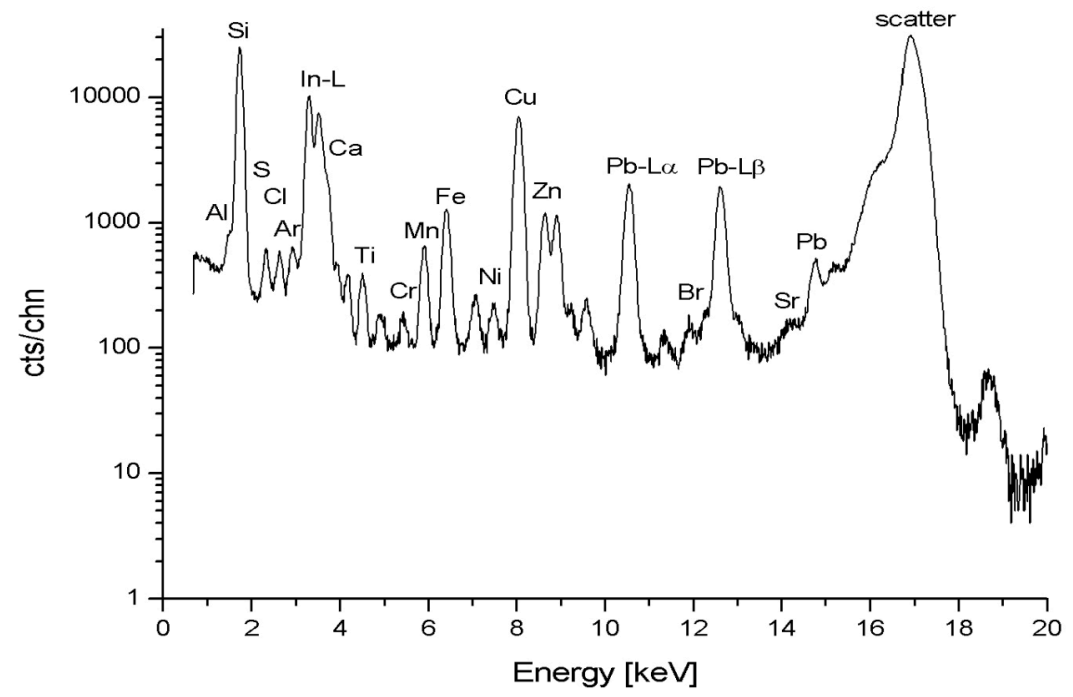

Fig.4 Spectrum of an aerosol sample sampled on a Si wafer at impactor stage 1, which corresponds to particles with a aerodynamic diameter smaller than $60 \mathrm{~nm} .1 \mathrm{~h}$ sampling, excitation energy: $17 \mathrm{keV}$ using the multilayer monochromator, $100 \mathrm{~s}$ measuring time.

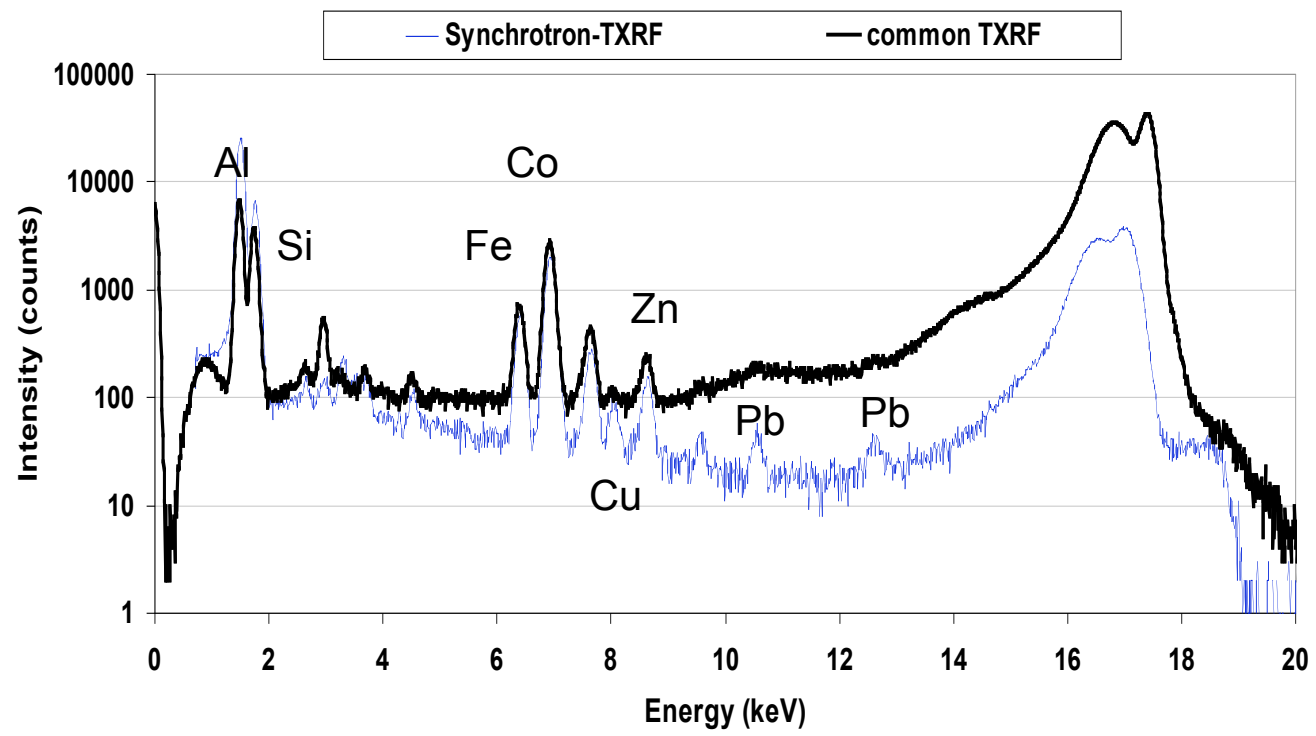

Fig 5. Comparison of A12O3, measuring with laboratory TXRF ( 8030C) and SRTXRF. Spectrum 


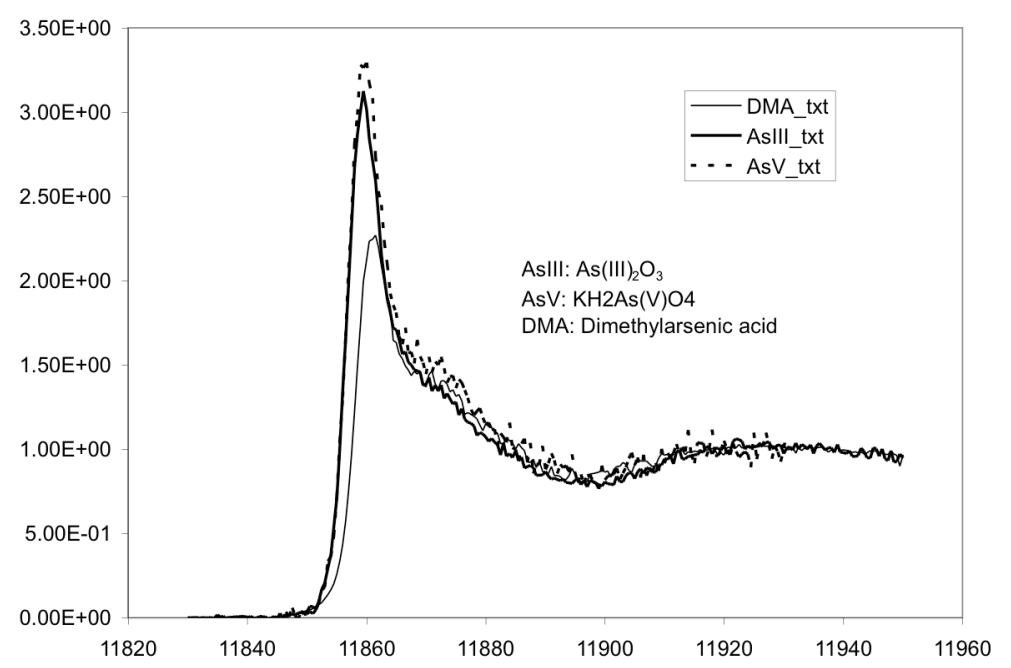

Fig. 6 XANES spectra of xylem sap from As III and As V fed plants in comparison to organic standard (DMA.. dimethylarsenic acid)

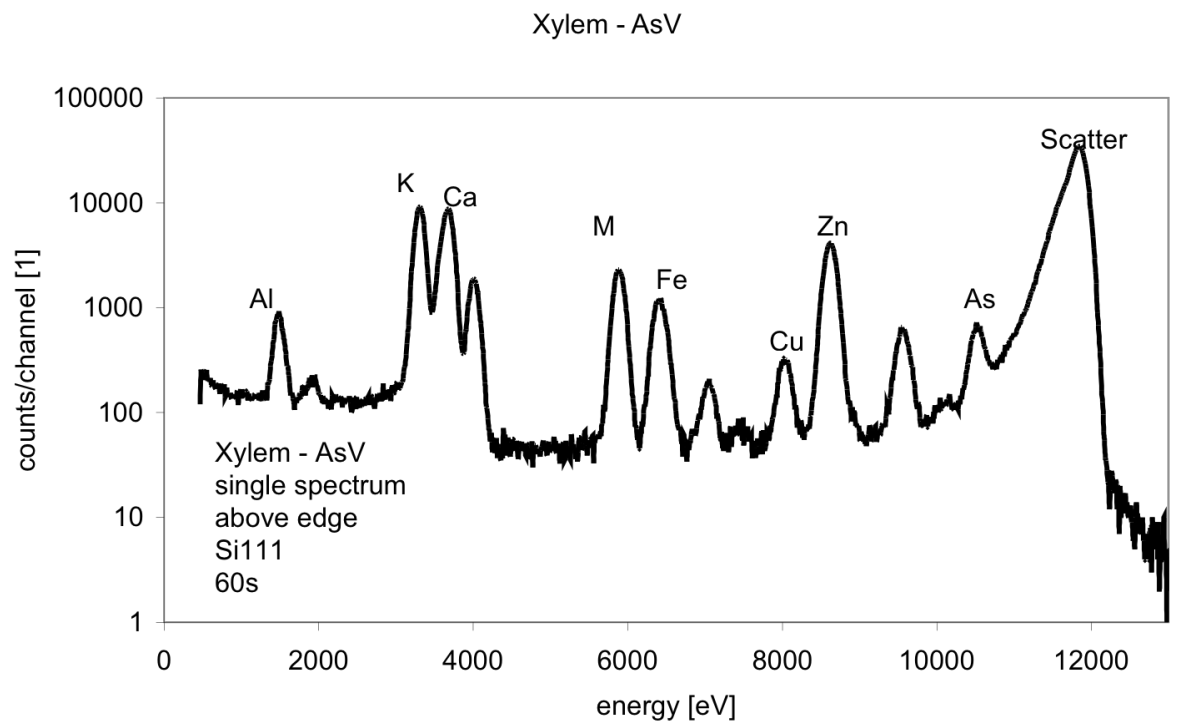

Fig. 7 Spectrum obtained during XANES scan from xylem sap. The As concentration was about $20 \mathrm{ppb}$, the detection limits in the range of $170 \mathrm{ppt}$ for As. 